\title{
DETERMINACIÓN DEL IMPACTO SOCIOECONÓMICO DE LAS POLÍTICAS DE INCLUSIÓN FINANCIERA EN EL MUNICIPIO DE MONTERÍA
}

\section{DETERMINATION OF THE SOCIO-ECONOMIC IMPACT OF FINANCIAL INCLUSION POLICIES IN THE MUNICIPALITY OF MONTERIA (COLOMBIA)}

\author{
Manuel Antonio Pérez-Vásquez ${ }^{1}$ y Francia Helena Prieto-Baldovino²
}

${ }^{1}$ Universidad del Sinú Elías Bechara Zainum, Colombia.Email: manuelperezv @unisinu.edu.co

${ }^{2}$ Universidad del Sinú Elías Bechara Zainum, Colombia.Email: franciaprieto@unisinu.edu.co

Para citar este artículo: Pérez, V. M. y Prieto, B. F. (2019). Determinación del impacto socioeconómico de las políticas de inclusión financiera en el municipio de Montería. Clío América, 13(26), 350-361. doi: http://dx.doi.org/10.21676/23897848.3577

Recibido: 17 junio de 2019

Aceptado: 08 de octubre de 2019

Publicado en línea: noviembre 28 de 2019

Palabras clave: impacto socioeconómic o; inclusión financiera; políticas.

JEL: D14; E61; G28.

\section{RESUMEN}

El presente artículo tiene como propósito central, determinar cuál es el impacto de las políticas de inclusión financiera en la ciudad de Montería. De allí, la importancia de hacer una exposición de los factores que vienen incidiendo en el cumplimiento de estas políticas en el municipio de Montería. La metodología de esta investigación empírica, hace énfasis, en un trabajo de campo que privilegió las fuentes de tipo primaria, con apoyo estadístico, mediante un análisis de tipo correlacional (regresión lineal simple). Los resultados proporcionan evidencia empírica, de que los sectores poblacionales de menores ingresos en Montería tienen menores posibilidades de acceso al campo financiero, debido a que no poseen garantías suficientes para cumplir con el pago de dichos créditos; en ese sentido, se requiere mayor inclusión financiera hacia los hogares de los sectores populares en dicho municipio. A partir de estos hallazgos, se concluye, que una mayor inclusión financiera para el municipio de Montería será favorable para el desarrollo de más acciones emprendedoras que activen la economía local y con los que se logre obtener mayores niveles de bienestar en la población monteriana.

\section{ABSTRACT}

The main purpose of this article is to determine what is the impact of financial inclusion policies in the city of Monteria

Keywords: financial inclusion; policies; socioeconom ic impact. (Colombia). Hence; the importance of exposing the factors that have been influencing compliance with these policies in the municipality of Monteria. The methodology of this empirical research emphasizes a field work that favored the application of primary sources, with statistical support, through a correlational analysis (simple linear regression), in order to identify which are the variables of greater incidence for the financial inclusion of the population of popular strata in Monteria. The results provide empirical evidence that the lower-income population sectors in Monteria have less possibilities of financial access, because they do not have sufficient guarantees to meet the payment of said credits; in this sense, greater financial inclusion is required towards the homes of the popular sectors in said municipality. Based on these findings, it is concluded that a greater financial inclusion for the municipality of Monteria will favor the development of more entrepreneurial actions that activate the local economy and achieve higher levels of well-being in the Monteriana population. 


\section{DETERMINACIÓN DEL IMPACTO SOCIOECONÓMICO DE LAS POLÍTICAS DE INCLUSIÓN FINANCIERA EN EL MUNICIPIO DE MONTERÍA}

\section{INTRODUCCIÓN}

El estudio tiene como propósito central determinar cuál es el impacto socioeconómico de las políticas de inclusión financiera en el municipio de Montería; por tanto, el artículo se centra en mostrar una realidad social que se hace cada día más compleja y en donde lo prioritario es que la inclusión financiera esté orientada hacia la mejora de las condiciones socioeconómicas de una gran población colombiana, que requiere mayor orientación en cuanto al uso de los productos y servicios que ofrecen las instituciones financieras (Cano, Esguerra, García, Rueda y Velasco, 2014).

En Colombia la inclusión financiera tiene dificultades para su desarrollo, debido a las connotaciones socioeconómicas, educacionales y etarias de la población, y en parte a la falta de conocimiento y la desconfianza que existe en el sector financiero para expedir créditos a los sectores más vulnerables del país. Por otra parte, los hogares que presentan menores ingresos son menos atractivos para el sector financiero en el otorgamiento de un crédito, debido a que no cuentan con un buen respaldo económico para que se garantice la devolución de dicho préstamo en un período determinado (Salas, 2015).

En el presente artículo se plantea como interrogante de investigación establecer cuál es el impacto socioeconómico de las políticas de inclusión financiera en el municipio de Montería. Por tanto, es de importancia poder determinar si estas políticas están siendo favorables en los hogares más populares en el municipio de Montería, departamento de Córdoba. En el desarrollo del artículo se hace una exposición teórica sobre la inclusión financiera, dando connotación particular a las políticas públicas de inclusión financiera en Colombia, y luego el abordaje acerca de la Banca de Oportunidades y la Superintendencia Financiera de Colombia, por ser estas entidades un apoyo institucional a tales políticas en el país. De igual manera, al final se espera que los resultados obtenidos sirvan de referente investigativo para que se profundice en mayor grado acerca de esta situación financiera que afecta la composición del gasto y del ahorro de la población colombiana. El estudio tiene como límites comunes, dentro del proceso de investigación, la escasez de datos o de estudios referentes a la inclusión financiera a nivel local o nacional.

El artículo es de tipo descriptivo, evidenciando los resultados de una investigación empírica que se realizó a través de la aplicación de una encuesta dirigida a los hogares de los estratos 1 y 2 en la ciudad de Montería en el año 2017. En el procedimiento se desarrolló el método correlacional de las variables objeto de estudio (crédito, sistema financiero, transacción, medios de pago) y posteriormente se efectuó un contraste unifactorial de los datos obtenidos (regresión lineal simple) con el objeto de identificar las especificidades del fenómeno analizado, que en este caso está referido a las políticas de inclusión financiera en los hogares más vulnerables del municipio de Montería. Se espera que los hallazgos sirvan de fundamento para plantear alternativas de solución en el logro de una mayor inclusión financiera en los hogares situados en los sectores populares de la ciudad de Montería, debido a que estos no poseen garantías suficientes para cumplir con el pago de los créditos bancarios ni hacer ahorro o inversión de tipo productivo.

\section{Inclusión financiera}

La inclusión financiera ha sido abordada desde diversos enfoques por diferentes autores. Según Bayero (2015), la inclusión financiera ocurre cuando una persona o empresa no tiene la capacidad de adquirir productos financieros adecuados. Por su parte, Allen, Demirgüç-Kunt, Klapper y Martínez (2016) definen que la inclusión financiera es el uso de los servicios financieros dentro de lo formal. Para Appleyard (2011), la inclusión financiera está referida a las personas y las organizaciones que pueden hacer apertura de una cuenta bancaria y obtener fácil acceso a los productos financieros que ofrece la banca.

El Committee on Financial Inclusion, CFI (2008) establece una definición un poco más compleja, al exponer que es el proceso en donde se garantizan los préstamos o créditos de una manera eficaz, cuando lo soliciten los sectores populares a un costo que es factible. Para Thorat (2006), este factor se traduce en la asequibilidad de servicios financieros por parte de las personas, hogares o empresas.

Algunos autores exponen que la inclusión financiera tiene como propósito central que se pueda brindar toda una serie de productos y servicios financieros a la población en general, en términos de asequibilidad, oportunidad y pertinencia; al respecto se considera que:

El proceso de promoción de un acceso asequible, oportuno y adecuado a una amplia gama de productos y servicios financieros regulados y la ampliación de su uso por todos los segmentos de la sociedad, a través de la aplicación de enfoques innovadores o existentes hechos a la medida, incluyendo actividades de sensibilización y de educación financiera, con el fin de promover el bienestar económico y la inclusión económica y social (García, Grifoni, López y Mejía, 2013, p. 
22).

En línea con lo anterior, la inclusión financiera se identifica con la creación de las políticas financieras que obligan la creación de productos y servicios bancarios accesibles para todos (Bernad, Fuentelsaz y Gómez, 2008), y que tienen como objetivo facilitar el acceso a los servicios bancarios, persiguiendo la creación de oportunidades monetarias para reducir la desigualdad social y la pobreza (Chibba, 2009).

A nivel mundial, se ha concebido que la inclusión financiera es favorable al crecimiento económico, debido a que genera mayores colocaciones de dinero en el público, para que se realicen más inversiones al sector productivo, y ello lleva a mayores tasas salariales de ingresos. En el caso de los hogares, se busca que el otorgamiento de créditos para pequeños negocios sea una ayuda eficaz en función de que estas familias se liberen de la pobreza (Solo, 2008). Según Vera y Titelman (2013), "Un requisito para que los sistemas financieros sean funcionales a un desarrollo económico y social sostenible es el de la inclusividad” (p. 9). Según la Comisión Multisectorial de Inclusión Financiera, CMIF (2015), "Las políticas de inclusión financiera son herramientas promotoras de la inclusión social y el desarrollo económico" (p. 59).

En cuanto a la no inclusión financiera de los sectores más vulnerables, el Global Financial Development Report, GFD (2013) expone que "la no inclusión financiera está referida a las Pymes y aquellas personas que presentan menores ingresos, por tanto, tal situación de debilidad económica les dificulta el acceso a servicios financieros" (p. 26), y a la vez se refuerzan las desigualdades sociales debido a que una gran mayoría no puede contar con recursos de capital para emprendimiento, mientras que otros sí poseen recursos propios para todo.

Ante la necesidad de que se incluya a la población de menores ingresos dentro del contexto de los sistemas bancarios o de financiación, surgen los programas de inclusión financiera a nivel mundial, debido a que estos son parte de las estrategias de las naciones en función de la reducción de la pobreza y la vulnerabilidad en cada región. Lo que se pretende con estas políticas o programas institucionales es que los productos financieros de la banca privada o pública lleguen con facilidad a los estratos más bajos en los países, y ello a su vez se convierta en un impulsor de la inversión, aumenten las capacidades empresariales, se generen mayores ingresos en el público o los hogares, y como consecuencia de todo ello se logren mejores condiciones de vida (Chakravarty y Pal, 2013). En el año 2006 el United Nations Committee on Building Inclusive Financial Sectors solicitó a los bancos centrales $\mathrm{y}$ a nivel estatal de las naciones que tengan como eje central la inclusión financiera dentro de sus políticas financieras, y considerando los términos de estabilidad y regulación prudencial, lográndose así mayor seguridad en el establecimiento de los fondos financieros (Kumar, Narain y Rubbani, 2015). En ese sentido, Inglaterra ha generado un fondo de inclusión financiera orientado a la promoción de dicha estrategia y se han definido responsabilidades a la banca y las cooperativas de crédito para que se desarrolle en favor de los más necesitados (Leeladhar, 2006). En Estados Unidos, a través de la Ley de Reinversión Comunitaria se estableció que los bancos y/o instituciones de ahorro extendieran sus créditos a sectores de ingresos bajos y medios en dicho país.

\section{Políticas públicas de inclusión financiera en Colombia}

En Colombia la política pública ha estado fortaleciendo mediante los planes nacionales de desarrollo, lineamientos estratégicos para que haya mayor inclusión financiera en la población de menores recursos del país; en ese sentido, la expedición de la Ley 1511 de 2007 (Plan Nacional de Desarrollo 2006-2010) estableció una política a largo plazo dirigida a crear las condiciones necesarias para facilitar el acceso y uso de servicios financieros integrales a la población colombiana, y a que se fortalezca la institucionalidad de la Banca de Oportunidades. Por su parte, la Ley 1540 de 2011 (Plan Nacional de Desarrollo 2010-2014) adoptó como una meta concreta la inclusión financiera, y para ello se promulgaron medidas en función de garantizar la oferta en regiones apartadas, diseñar productos adecuados para los diferentes segmentos poblacionales y promover la educación económica y financiera. Finalmente, la Ley 1753 de 2015 (Plan Nacional de Desarrollo 2014-2018) establece la necesidad de que se complementen acciones de monitorización y seguimiento sobre la inclusión financiera en el país.

Con relación a la situación financiera en Colombia, se pone en relevancia que los indicadores a nivel nacional ha evidenciado un notable crecimiento en el sector financiero, tal como lo evidencia el nivel de bancarización del país y el correspondiente uso de los productos financieros de dicho sector, que señala que la población adulta del país presenta al menos un producto financiero. Según la participación porcentual de entrada financiera en el país, en el año 2017 ingresaron 1,1 millones al sistema financiero, y $77,8 \%$ de esa población ingresó a través de cuentas de ahorro, $4 \%$ ingresó a través de créditos de consumo, 7,2\% por concepto de microcrédito y $6,9 \%$ ingresó por tarjetas de crédito (Castaño, 2018). Por otra parte, el Banco Mundial entre los años 2011 y 2014 presenta su reporte financiero indicando que el porcentaje de colombianos que tienen una cuenta en una institución financiera pasó de 30 $\%$ a $38 \%$. Esta mejoría toma mayor significancia entre los 
colombianos de menores ingresos, donde este porcentaje pasó de $13 \%$ a $23 \%$. Sin embargo, la formalización sigue siendo parte de la brecha en la canalización de recursos financieros por parte de la banca. En 2014 solo $28 \%$ de las personas que ahorraron mencionaban haberlo hecho en el sistema financiero formal (Demirguc-Kunt, Klapper, Singer y Van Oudheusden, 2014). También entre los años 2011 y 2014 el porcentaje de empresas que tenían al menos un producto financiero creció significativamente a $36 \%$. En 2014, cerca de $50 \%$ de la cartera total del sistema financiero se destinaba a las pequeñas y medianas empresas (PYMES) (Organización para la Cooperación y el Desarrollo Económico, OECD, 2016).

Los anteriores datos, en conjunción con las políticas públicas del país ya mencionadas, dan cuenta de que el Estado colombiano ha hecho un esfuerzo institucional con el objeto de promover el acceso y uso de productos financieros por parte de la población más necesitada, y como parte de las estrategias de educación financiera y protección al consumidor, tal como está contenido en la normatividad al respecto. Sin embargo, aún la realidad del país muestra un panorama de preocupación, debido a que estas políticas no han tenido la eficacia necesaria en cuanto a poder brindar una mayor cobertura y fácil acceso de los productos y servicios financieros en la población más vulnerable, y en consideración a que el sistema financiero influye de manera significativa en el progreso económico de las regiones del mundo, en especial en países menos desarrollados, como el caso colombiano; en ese sentido, la banca debe ser colaborativa con el bienestar económico del país. De lo anterior se infiere la necesidad de que haya inclusión financiera en aquellos sectores vulnerables de la sociedad (Roa, 2013).

\section{La Banca de Oportunidades y la Superintendencia Financiera de Colombia}

En Colombia el gobierno nacional, en línea con las recomendaciones contenidas en el documento del Consejo Nacional de Política Económica y Social, CONPES 3424 de 2006, expidió el Decreto 3078 de 2006, el cual instauró el Programa de Inversión llamado Banca de Oportunidades (BdO) y cuyo objetivo central fue crear las condiciones necesarias para promover el acceso al crédito y los demás servicios financieros de la población de menores ingresos, las Mipymes y emprendedores (Decreto 3078, 2006, art. 1, como se citó en Rodríguez-Lozano, 2017). La Superintendencia Financiera de Colombia-SFC- publica de manera anual los reportes de inclusión financiera. Allí se expone que:

En los últimos años, la inclusión financiera ha adquirido gran importancia a nivel mundial, tanto en las agendas de gobierno, especialmente de países en vía de desarrollo, como a través de iniciativas multilaterales, reconociéndose de este modo la importancia de lograr una mayor inclusión financiera como herramienta de lucha contra la pobreza, así como para una mejor distribución de ingresos y el fomento del desarrollo económico (p. $9)$.

Según la BdO y la SFC (2012), la inclusión financiera es "el acceso y utilización de servicios financieros formales por parte de las actividades económicas y de la población" (p. 14). Las políticas de inclusión financiera son favorables a la población vulnerable, y en ese sentido, Cecchetti, Flores y Krause (como se citó en Cano et al., 2014) consideran que:

El mejor acceso al mercado de crédito permite una reducción en las restricciones de liquidez de los hogares, lo que se traduce en un crecimiento mucho más estable del consumo y en una moderación de los ciclos reales de la economía, favoreciendo el comportamiento de los agregados económicos (p. 4).

En línea con lo anterior, el gobierno colombiano ha promovido, mediante las políticas públicas en materia de inclusión financiera, el que haya un mayor acceso a los productos financieros por parte de los sectores más marginados de la sociedad, debido a que la inclusión financiera contribuye en la acumulación de capital de la economía (Cano et al., 2014).

\section{METODOLOGÍA}

El presente artículo es de tipo descriptivo, evidenciando los resultados de una investigación empírica con base en la recolección de información cuantitativa, a través de la aplicación de una encuesta con preguntas cerradas y dirigida a los hogares de los estratos 1 y 2 en la ciudad de Montería en el año 2017. Se aplicó el método de tipo correlacional, en el cual se trata de determinar el grado de relación entre las variables objeto de estudio (crédito, sistema financiero, transacción, medios de pago), para luego realizar una contrastación unifactorial de los datos obtenidos (regresión lineal simple), para con ello establecer su significancia, especificidades y correlación de los mismos. Se pretende validar si la composición socioeconómica de los hogares objeto de estudio es un factor que incide desfavorablemente en la inclusión financiera en el municipio de Montería. 


\section{RESULTADOS}

Según el Boletín Estadístico - Contexto Sectorial Montería en el departamento de Córdoba para el año 2017 en la ciudad de Montería se calcula aproximadamente unos 84 015 hogares, y aplicando un muestreo arbitrario aleatorio de $1 \%$ a dicha población, se obtuvo una muestra representativa de 840 hogares por encuestar. A continuación se muestran los resultados de un trabajo de campo efectuado en los hogares de los estratos 1 y 2 de la ciudad de Montería, en el cual se analizan las variables de mayor impacto socioeconómico y las políticas de inclusión financiera en los hogares. En el estudio se describe que la mayoría de los hogares encuestados tienen ingresos inferiores a un millón y medio de pesos, y por tanto, es común que esta situación dificulte la obtención de crédito por parte de los hogares, tal como se refleja a continuación, en la Tabla 1.

Tabla 1. Crédito en el Sistema Financiero

\begin{tabular}{|l|l|}
\hline 1. ¿Tienen o tuvieron algún crédito del sistema financiero? & \\
\hline Ninguno & 468 \\
\hline Tarjeta de crédito & 89 \\
\hline Microcrédito & 70 \\
\hline Educativo & 48 \\
\hline Vivienda & 5 \\
\hline Hipoteca & 10 \\
\hline Comercial & 0 \\
\hline Con una ONG & 5 \\
\hline Para víctimas del conflicto armado & 145 \\
\hline Total & 840 \\
\hline
\end{tabular}

Fuente: elaboración propia.

La figura 1, muestra, que 468 personas, que representan 55 $\%$ de los encuestados, manifiestan que no han tenido ningún crédito del sistema financiero. Este resultado es típico, si se considera las dificultades para el acceso al crédito de los usuarios de estratos populares de la ciudad de Montería.

Figura 1. Crédito en el Sistema Financiero

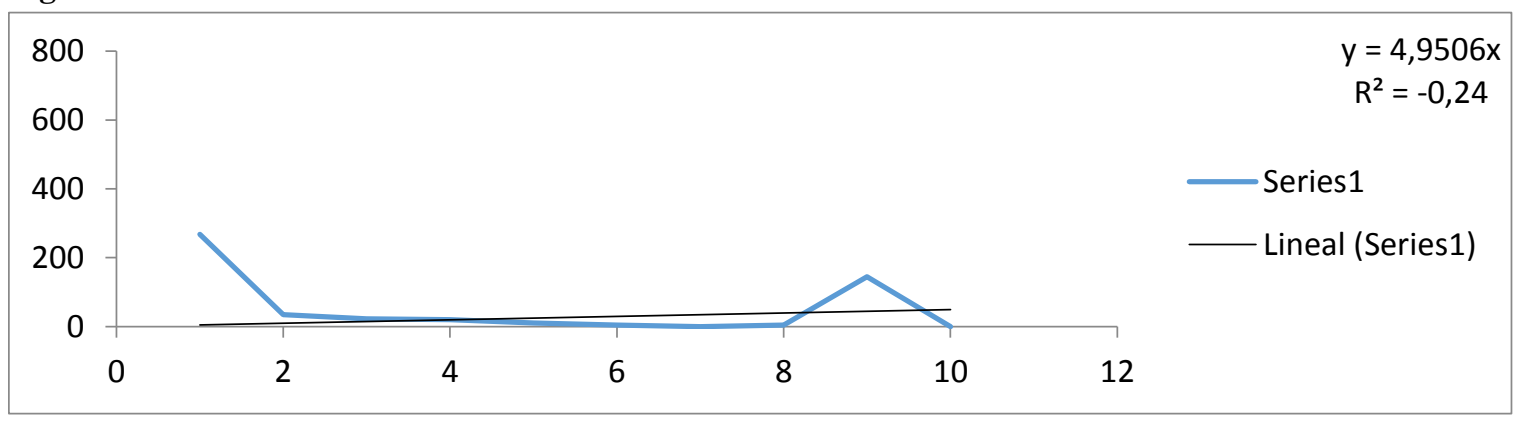

Fuente: elaboración propia.

Los tipos de transacciones realizados por los hogares encuestado (mostrado en la Tabla 2), evidencian, de que la cuenta corriente, tiene mayor relevancia que la cuenta de ahorro, lo cual, tal situación podría obedecer, a que el público desea tener una cuenta que refleje un mayor movimiento monetario, y tal condición, sirva de garantía para lograr prestamos significativos del sector bancario. A continuación, se exponen, en la Tabla 2, las preferencias de las transacciones bancarias por parte, de los hogares encuestados. 
Tabla 2. Transacción realizada

\begin{tabular}{|l|l|}
\hline 2. ¿Qué tipo de transacción realiza o utiliza? & \\
\hline Cuenta de ahorros & 266 \\
\hline Cuenta corriente & 495 \\
\hline Cuenta de ahorro programado & 78 \\
\hline CDT & 1 \\
\hline Aportes sociales & 0 \\
\hline Total & 840 \\
\hline
\end{tabular}

Fuente: elaboración propia.

La figura 2 muestra, que $59 \%$ de las personas encuestadas, manifiestan que hacen transacción bancaria por cuenta corriente, y solo $31 \%$ pone de manifiesto que tiene cuenta de ahorros. Este resultado es atípico, ya que se considera más asequible la cuenta corriente para acceder a créditos financieros, pero a pesar de ello se dificulta su acceso a los usuarios de estratos populares de la ciudad.

Figura 2. Transacción realizada

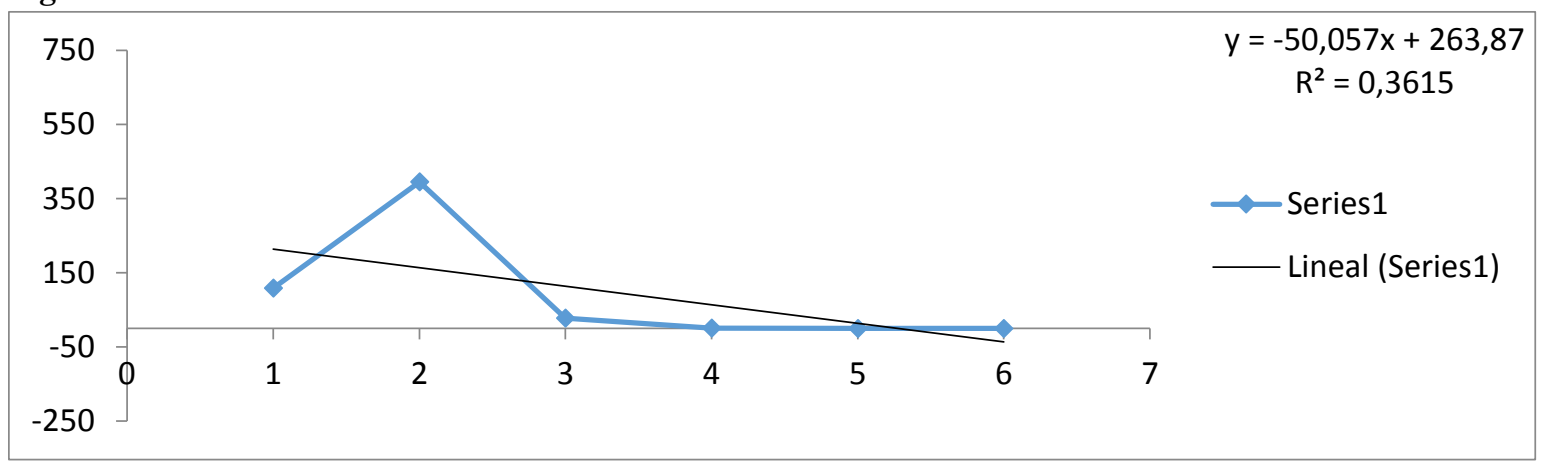

Fuente: elaboración propia.

Con relación a los medios más usados por parte de los hogares encuestados para efectuar transacciones monetarias, Efecty y Baloto son los de mayor interés en el público, debido a que ofrecen mayor seguridad $\mathrm{y}$ disponibilidad para los usuarios, y ello puede comprobarse a continuación en la Tabla 3.

Tabla 3. Medio más usado para transacciones

\begin{tabular}{|l|l|}
\hline 3. ¿Cuál es el medio más usado para sus transacciones? & \\
\hline Oficinas & 180 \\
\hline Cajeros automáticos & 150 \\
\hline Datáfono & 3 \\
\hline Por teléfono & 0 \\
\hline Internet & 0 \\
\hline Débito automático & 0 \\
\hline Corresponsal bancario & 0 \\
\hline Efecty o Baloto & 502 \\
\hline Otro & 5 \\
\hline Total & 840 \\
\hline
\end{tabular}

Fuente: elaboración propia.

La figura 3, indica que la mayoría de los jefes de hogares encuestados, manifiestan que Efecty y Baloto son los medios preferidos para realizar transacciones. Este resultado, confirma el hecho de que los hogares monterianos, solo hacen operaciones pequeñas de transferencia monetaria, y obviando al sistema bancario 
para las mismas.

Figura 3. Medio más usado para transacciones

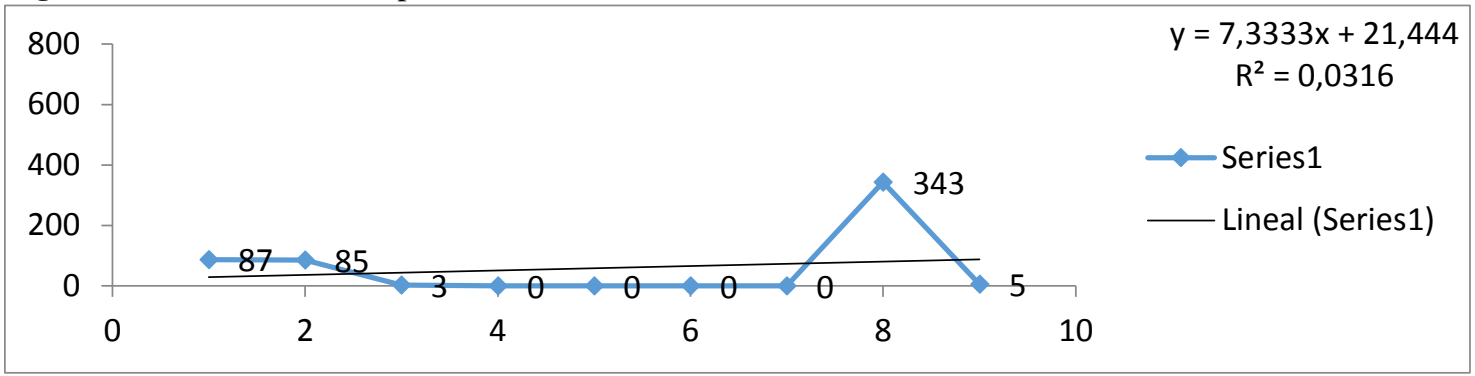

Fuente: elaboración propia.

De los hogares encuestados, $77 \%$ opinan que la transacción que más realizan son los pagos de servicios públicos. Y este resultado, está en concordancia con la

anterior respuesta, debido a que Efecty y Baloto son las cajas referidas para hacer dichos pagos, como lo muestra la Tabla 4.

Tabla 4. Transacción que más realiza

\begin{tabular}{|l|l|}
\hline 4. ¿Cuál es la transacción que más realiza en el medio seleccionado? & \\
\hline Pagos (servicios públicos) & 650 \\
\hline Retiros & 170 \\
\hline Transferencias & 10 \\
\hline Depósitos & 6 \\
\hline Transacciones internacionales & 2 \\
\hline Consulta de saldo & 2 \\
\hline Total & 840 \\
\hline
\end{tabular}

Fuente: elaboración propia.

La figura 4 corrobora el hecho de que las transacciones que más hacen los hogares son los pagos de servicios públicos, debido a que estos son los de mayor urgencia dentro de las diversas prioridades del gasto familiar.

Figura 4. Transacción que más realiza

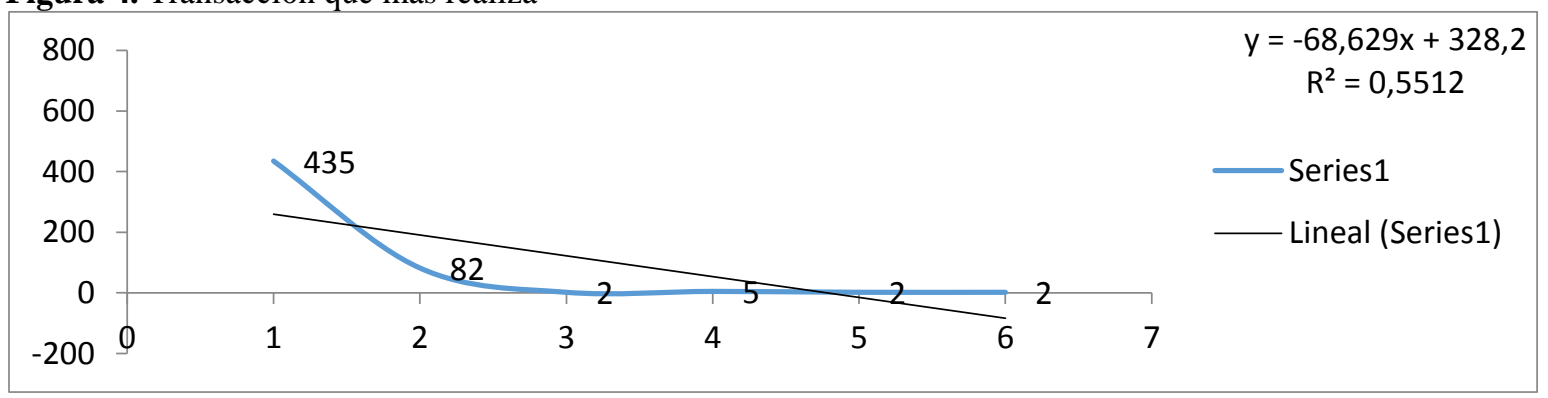

Fuente: elaboración propia.

También se observó, que la mayoría de los encuestados no reciben por ningún medio depósito internacional. Este resultado obedece a las limitaciones económicas de estos hogares que no cuentan con ningún apoyo económico del exterior ni ejercen actividades que impliquen transferencias monetarias de un país a otro.

\section{Análisis de los resultados}

Los hallazgos, con base en el análisis estadístico de los resultados de la encuesta, evidenciaron que la composición socioeconómica de los hogares de estratos 1 y 2 en el municipio de Montería es un factor que incide desfavorablemente en la inclusión financiera, debido a la menor capacidad de ingresos y el menor uso de los servicios financieros ofrecidos por la banca privada y la pública, y en razón de que estos hogares no cuentan con 
garantías suficientes para cumplir con el pago de dichos créditos. En ese sentido, se corrobora, con lo expuesto por el Global Financial Development Report, GFD (2013), que a las personas que presentan menores ingresos debido a su situación de vulnerabilidad económica, se les dificulta el acceso a servicios financieros. En ese orden de ideas, se requiere que los productos y servicios bancarios sean más asequibles al público (Bernad, Fuentelsaz y Gómez, 2008), y generen oportunidades monetarias para reducir la desigualdad social y la pobreza (Chibba, 2009).

Por otra parte, un porcentaje significativo de jefes de hogares de estratos 1 y 2 de la ciudad de Montería manifiestan que no han recibido préstamos de tipo formal en atención a un gasto en el hogar, o por una urgencia de tipo económico. Además de ello, la mayoría de los encuestados expresan que hacen muy poco algún tipo de transacción financiera o bancaria, y que se considera más común la cuenta corriente que la de ahorros. Este resultado da a conocer la necesidad de que haya inclusión financiera en estos hogares, y ello implica que haya mayor información sobre los préstamos otorgados por el sistema financiero, específicamente de la banca privada. Por otra parte, la mayoría de los jefes de hogar manifiestan que Efecty (plataforma de pagos especializada en recaudos, pagos y giros a nivel nacional) y Baloto (empresa que ofrece otros servicios como recargas, pagos, retiros y giros que pueden realizarse en todo el país) son los medios preferidos para hacer transacciones. Esto indica que tales transacciones obedecen al cumplimiento de pagos por vía de servicios públicos, obviando al sistema bancario para la realización de depósitos y retiros. De igual manera, los encuestados señalan que las transacciones monetarias que hacen son solo de tipo local y solicitan préstamos para gastos del hogar, o para el pago de otro crédito, por lo cual se deduce que son nulos los préstamos para inversión en negocios. En cuanto a la correlación lineal, esta indica que los resultados corroboran la linealidad de los datos, es decir, las variables se asocian de manera lineal, dando mayor veracidad a los datos obtenidos en el trabajo de campo.

\section{DISCUSIÓN}

Con relación a identificar cuál es el impacto socioeconómico de las políticas de inclusión financiera en el municipio de Montería, la aplicación de la encuesta constata, que la composición socioeconómica de los hogares de estratos 1 y 2 en el municipio de Montería incide desfavorablemente en la inclusión financiera de estas familias, debido a que estas no poseen la capacidad de ingreso necesaria para poder garantizar el cumplimiento de los pagos de dichos créditos. Esta realidad es coincidente con la teorización en materia de inclusión financiera a nivel general, y en particular, en lo que ocurre en la población de menores ingresos en el país, que tiene cada vez menor acceso a los productos y servicios del sector financiero.

Por otra parte, se indica, que el contenido teóricoconceptual de la investigación, corrobora los resultados de la encuesta, al constatarse, que sectores poblacionales de menores ingresos en las ciudades, tienen menores posibilidades de acceso al campo financiero, ante ello ha habido avances significativos en materia de inclusión financiera en Colombia, es decir, se han implementado políticas financieras de inclusión a nivel nacional, las cuales han sido lideradas por las Superintendencia Financiera y la Banca de Oportunidades. Sin embargo, aunque el gobierno colombiano, ha dictaminado una serie de normas (Leyes 1511 de 2007, 1540 de 2011 y 1753 de 2015), en función de que haya mayor inclusión financiera y de mayores esfuerzos institucionales (Banca de Oportunidades y la Superintendencia Financiera de Colombia), aún no se logra la cobertura necesaria para que personas y pequeños negocios tengan posibilidades de crédito y de ahorro. En ese sentido, es imperativo que se evalué a nivel de las políticas públicas si verdaderamente los mecanismos de instrumentación de tales políticas están siendo eficaces para favorecer a los hogares más vulnerables en Colombia, a través de una mayor cobertura de la inclusión financiera, y como un caso muy similar a lo que ocurre en el municipio de Montería, en donde la inclusión financiera; sigue siendo un propósito aún por materializar, y en función de dinamizar la economía a nivel local.

Los resultados del trabajo de campo muestran que aún se tiene dificultad el acceso y uso de productos financieros (banca privada nacional y de las trasnacionales), en los hogares en el municipio de Montería. Según los datos de la encuesta, estas familias dirigen sus ingresos solo a cubrir necesidades de pago u obligaciones, y no a operaciones de negocios o de capitalización del dinero en el tiempo. Tal situación no valida los postulados teóricos que exponen, acerca de que la inclusión financiera es colaborativa con el ingreso de las poblaciones más vulnerables y, por ende, no aporta al crecimiento económico de las naciones más pobres.

Por otra parte, los resultados obtenidos en la investigación ponen en evidencia dos aspectos centrales: uno, el hecho de que comúnmente la mayoría de los hogares de la ciudad de Montería no reciben préstamos de tipo formal del sector bancario para cubrir un gasto en el hogar o para atender una 
urgencia de tipo económico. De igual manera, hay poco uso de algún tipo de transacción financiera o bancaria, debido a la desconfianza que existe con relación a que una entidad financiera sea el guarda de sus dineros. Lo anterior ha llevado a que exista la necesidad de que se elaboren propuestas estratégicas para una mayor inclusión financiera para el municipio de Montería, debido a que un mayor acceso a los productos financieros será favorable para el desarrollo de más acciones emprendedoras que activen la economía local y se logre mayores niveles de bienestar en la población monteriana, en línea con la fundamentación teórica sobre la inclusión financiera y en razón de los lineamientos contenidos en las políticas financieras del país en lo que respecta a facilitar un mayor acceso financiero a las comunidades más pobres.

\section{CONCLUSION}

La situación de vulnerabilidad económica de muchas familias colombianas impide el fácil acceso a los productos y servicios de la banca pública y privada en el país, debido a que estos hogares no cumplen con las garantías impuestas por estas instituciones financieras para el pago de créditos libres o en el cumplimiento de los préstamos para capitalización, y en lo que respecta al uso de los portafolios de servicios financieros; esta realidad coincide con la revisión de la literatura en materia de inclusión financiera, en donde se corrobora que a las poblaciones de menores ingresos en el mundo se les dificulta el acceso a los servicios bancarios. En ese orden de ideas, es de importancia que haya esfuerzos interinstitucionales y declaraciones normativas en función de que las naciones más pobres puedan lograr una mayor inclusión financiera; esto aunado con tales propósitos constituyen herramientas o mecanismos de lucha contra la pobreza.

Con relación al objetivo central de determinar el impacto socioeconómico de las políticas de inclusión financiera en el municipio de Montería, los resultados de la encuesta confirman que la composición socioeconómica de los hogares de estratos 1 y 2 del municipio de Montería incide desfavorablemente en la inclusión financiera de estas familias, debido a que estas no poseen la capacidad de ingreso necesaria para poder garantizar el cumplimiento de los pagos de dichos créditos. Esta realidad es coincidente con lo anteriormente expresado y con relación a la teorización en materia de inclusión financiera.

Se concluye que los sectores poblacionales de menores ingresos en el municipio de Montería tienen menores posibilidades de acceso al campo financiero, debido a la falta de garantías para el cumplimiento de los pagos y a la excesiva tramitología para la consecución de dichos préstamos, y por tanto, una mayor inclusión financiera en esta localidad permitirá que haya una mayor irrigación de los productos y servicios financieros hacia el desarrollo de actividades económicas de tipo formal e informal por parte de la población monteriana, y ello a su vez contribuye de manera significativa en la inversión, el ahorro y la obtención de capitales a menores costos de financiación.

La revisión de la literatura ha puesto en relevancia que la inclusión financiera puede ser un mecanismo colaborativo con la dinamización de la economía, debido a que coloca mayores recursos monetarios en las personas y pequeñas empresas, y por consiguiente se aumenta el gasto y la inversión, como demanda agregada en provecho del crecimiento económico y en favor del bienestar general de las poblaciones más pobres.

Se espera que esta investigación sea fundamento o motivación para que se desarrollen nuevos estudios que aborden esta temática desde un enfoque teóricoconceptual, y que particularmente estén orientados hacia el bienestar y el desarrollo económico en la región del Caribe colombiano.

\section{Declaración sobre conflictos de interés}

Declaramos que este artículo es independiente con respecto a las instituciones financiadoras y de apoyo, y que durante la ejecución del trabajo o la redacción del manuscrito no han incidido intereses o valores distintos a los que usualmente tiene la investigación; por tanto, no existe ningún conflicto de interés para su publicación.

\section{REFERENCIAS BIBLIOGRÁFICAS}

Allen, F.; Demirgüç-Kunt, A.; Klapper, L., y Martínez, P. M. (2016). The Foundations of Financial Inclusion: Understanding Ownership and Use of Formal Accounts. Journal of Financial Intermediation, 27, 1-30. Recuperado de https://revistas.javeriana.edu.co/filesarticulos/CC/1845\%20(2017)/151555055004/.
Appleyard, L. (2011). Community Development Finance Institutions (CDFIs): Geographies of Financial Inclusion in the US and UK. Geoforum, 42(2), 250-258. Recuperado de https://revistas.javeriana.edu.co/files- articulos/CC/18- 
DETERMINACIÓN DEL IMPACTO SOCIOECONÓMICO DE LAS POLÍTICAS DE INCLUSIÓN FINANCIERA EN EL MUNICIPIO DE MONTERÍA

$\underline{45 \% 20(2017) / 151555055004 / .}$

Banca de las Oportunidades, $\mathrm{BdO}$ y Superintendencia Financiera de Colombia, SFC (2012). Reporte de inclusión financiera 2011. Bogotá: Banca de las Oportunidades, Bancoldex - Banco de Comercio Exterior de Colombia S.A., BdO y Superintendencia Financiera de Colombia, SFC. Recuperado de

https://www.superfinanciera.gov.co/descargas? $\underline{\text { com }=\text { institucional } \& \text { name }=\text { pubFile } 10009}$ 70\&downloadname $=18052012$ reportein clusion.pdf

Bayero, M. (2015). Effects of Cashless Economy Policy on Financial Inclusion in Nigeria: An Exploratory Study. Procedia - Social and Behavioral Sciences, 172, 49-56. Recuperado de https://revistas.javeriana.edu.co/filesarticulos/CC/18-45\%20 (2017)/151555055004/.

Bernad, C.; Fuenteslsaz, L., y Gómez, J. (2008). Deregulation and its long-run effects on the availability of banking services in low-income communities. Environment and Planning A., 40(7), 1681-1696. Recuperado de

https://intellectum.unisabana.edu.co/bitstream/h andle/10818/22874/Mar\%EDa\%20Luisa \%20Jaramillo\%20G\%F3mez\%20\%20(t esis).pdf?sequence $=1$

Comisión Multisectorial de Inclusión Financiera, CMIF (2015). Estrategia Nacional de Inclusión Financiera. Lima, Perú: Ministerio de Economía y Finanzas. Recuperado de https://revistas.javeriana.edu.co/filesarticulos/CC/1845\%20(2017)/151555055004/.

Committee on Financial Inclusion, CFI (2008). Report of the Committee on Financial Inclusion. New Delhi: Committee on
Financial Inclusion, CFI. Recuperado de https://revistas.javeriana.edu.co/filesarticulos/CC/18-45\%20 (2017)/151555055004/.

Chakravarty, S., \& Pal, R. (2013). Financial inclusion in India: An axiomatic approach. Journal of Policy Modeling, 35(5), 813-837. Recuperado de http://www.igidr.ac.in/pdf/publication/ WP-2010-003.pdf.

Cano, C.; Esguerra, M.; García, J.; Rueda, L., y Velasco, A. (2014). Inclusión financiera en Colombia. Banco de la República. Colombia. Recuperado de

http://repositorio.banrep.gov.co/bitstrea $\underline{\mathrm{m} / \mathrm{handle} / 20.500 .12134 / 6618 / 6 . \% 20 \text { Incl }}$ usi\%C3\%B3n\%20financiera\%20en\%20 Colombia.pdf? sequence $=2 \&$ isAllowed $=$ $\mathrm{y}$.

Cecchetti, S.: Flores, L., \& Krause, S. (2006). Financial Development, Consumption Smoothing, and the Reduced Volatility of Real Growth. Working Paper AEA. Recuperado de

https://www.banrep.gov.co/sites/default/ files/eventos/archivos/sem_357.pdf.

Castaño, G. (2018). Futuro de la Inclusión Financiera Hacia la innovación sostenible y responsable en el sector financiero. Superintendente Financiero. Cartagena de Indias. Recuperado de file:

///C:/Users/User/Downloads/20180718a somicrofinanzas\%20(1).pdf.

Departamento Nacional de Planeación (2006). Consejo Nacional de Política Económica y Social, Conpes 3424 de 2006. Colombia. Recuperado de https://revistas.javeriana.edu.co/filesarticulos/CC/1845\%20(2017)/151555055004/m. 
Chibba, M. (2009). Financial Inclusion, Poverty Reduction, and the Millennium Development Goals. European Journal of Development Research, 21(2), 213230.

Recuperado dehttps://www.banrep.gov.co/sites/defa ult/files/eventos/archivos/sem_357.pdf.

Demirguc-Kunt, A.; Klapper, L.; Singer, D., y Van Oudheusden, P. (2015). The Global Findex Database 2014: measuring financial inclusion around the world (English). Policy Research working paper; no. WPS 7255. Washington, D.C.: World Bank Group. Recuperado de https://www.asobancaria.com/wpcontent/uploads/Ensayos-sobreinclusion-04051727-04-2018.pdf

García, N.; Grifoni, A.; López, J., y Mejía, D. (2013). La educación financiera en América Latina y el Caribe. Situación actual y perspectivas. Caracas: Corporación Andina de Fomento, CAF, Banco de Desarrollo de América Latina. Recuperado $\underline{\text { de }}$ https://www.oecd.org/daf/fin/financialeducation/OECD_CAF_Financial_Educ ation_Latin_AmericaES.pdf

Global Financial Development, GFD (2013). Global Financial Development Report: Rethinking the Role of the State in Finance. Washington: International Bank for Reconstruction and Development, the World Bank. Recuperado de https://revistas.javeriana.edu.co/filesarticulos/CC/1845\%20(2017)/151555055004/.

Kumar, A.; Narain, S., \& Rubbani, S. (2015). World Bank Lending for Financial Inclusion: Lessons com Reviews of Select Projects. Washington: Independent Evaluation Group, e World Bank Group. Recuperado de https://revistas.javeriana.edu.co/files-
articulos/CC/18-45\%20

(2017)/151555055004/.

Leeladhar, V. (2006). Taking Banking Services to the Common Man - Financial Inclusion. Reserve Bank of India Bulletin, New Delhi. Recuperado de: https://revistas.javeriana.edu.co/filesarticulos/CC/18-45\%20 (2017)/151555055004/.

Organización para la Cooperación y el Desarrollo Económico, OECD (2016), Financing SMEs and Entrepreneurs 2016: An OECD Scoreboard. OECD Publishing, Paris. Recuperado de https://www.asobancaria.com/wpcontent/uploads/Ensayos-sobreinclusion-04051727-04-2018.pdf

Roa, M. (2013). Inclusión financiera en América Latina y el Caribe: acceso, uso y calidad. Boletín del Centro de Estudios Monetarios Latinoamericanos (CEMLA), 121-148. Recuperado de http://www.cemla.org/PDF/boletin/PUB _BOL_LIX03-01.pdf

Rodríguez-Lozano, G. (2017). Inclusión financiera en Colombia. Análisis de su evolución en términos de eficiencia relativa._Universidad Nacional de Colombia. Recuperado de https://revistas.javeriana.edu.co/filesarticulos/CC/18-45\%20 (2017)/151555055004/.

Salas, Q. (2015). Financiamiento del Sector Industrial de las MIPYMES en el Municipio de Sincelejo. Universidad Nacional de Colombia. Recuperado de

http://bdigital.unal.edu.co/49976/1/Hebe rto\%20Salas.2015.pdf

Solo, T. (2008). Financial Exclusion in Latin America - Or the Social Costs of Not Banking the Urban Poor. Environment and Urbanization, 20(1), 47-66. 
DETERMINACIÓN DEL IMPACTO SOCIOECONÓMICO DE LAS POLÍTICAS DE INCLUSIÓN FINANCIERA EN EL MUNICIPIO DE MONTERÍA

Recuperado de

https://revistas.javeriana.edu.co/files-

articulos/CC/18-45\%

20(2017)/151555055004/.

Thorat, U. (2006). Financial Inclusion and Millennium Goals. New Delhi: Reserve Bank of India. Recuperado de http://sustainabledfs.lbs.edu.ng/FI-

Resources/2006_Thorat.pdf

Vera, C., y Titelman, D. (2013). El sistema financiero en América Latina y el Caribe: una caracterización. Santiago de Chile: Comisión Económica para América Latina y el Caribe, CEPAL, Serie Financiamiento para el desarrollo. Recuperado de https://revistas.javeriana.edu.co/filesarticulos/CC/18-45\%20(2017)/151555055004/. 